\title{
Rhinofacial conidiobolomycosis, two cases in Mexican patients from rural and urban backgrounds
}

\author{
Jorge F. Moisés-Hernández, Carlos Santamaría-Saad*, Montserrat Reyes-Millan, and \\ Adriana Furlong-Murillo \\ Department of Otorhinolaryngology and Head and Neck Surgery, Hospital General de México, Mexico City, Mexico
}

\begin{abstract}
Conidiobolomycosis is a relatively uncommon disease in humans, caused by Conidiobolus spp fungi. It manifests itself in rural environments with tropical climate. It is frequently associated with certain degree of immunosuppression such as hematologic malignancies, diabetes mellitus, and/or chronic use of steroids. Treatment of the localized disease is simple, although diagnosis, due to its low incidence, can represent a challenge to many doctors. The objective of this case series is to help identify, diagnose, and treat this disease in a timely manner.
\end{abstract}

Key words: Rhinosinusitis. Conidiobolus. Entomophthoramycosis. Fungal infections. Cutaneous mycosis.

\section{Introduction}

Conidiobolomycosis, also known as rhinofacial entomophthoramycosis, is a fungal disease affecting the skin and mucous membranes, caused by fungi of the order Entomophthorales (Conidiobolus coronatus, Conidiobolus incongruous, and Conidiobolus lamprauges)'. It occurs mainly in tropical regions worldwide and is classically associated with rural environments and low socioeconomic status ${ }^{2}$. Localized disease is easily treated. However, atypical and systemic presentations, or in case of immunocompromised patients, carry a high degree of morbidity and mortality ${ }^{3,4}$. The presentation in the head and neck is most commonly found in the rhinofacial region, with the glabellar region being the mostly affected ${ }^{5}$. The importance lies in the rarity of its presentation, making diagnosis difficult, due to its low incidence and lack of knowledge by specialists. It should be integrated into the differential diagnosis of centrofacial, nasal, and paranasal sinus tumors. Biopsy of the affected tissue is the diagnostic study of choice with adequate histological processing'. We present two cases of confirmed infection by fungi of the species Zygomycota spp (C. coronatus) in non-immunocompromised Mexican patients, the first patient coming from a rural environment with tropical climate and the second patient hailing from an urban environment with no apparent classical background to contract the disease.

\section{Case study}

First case: A 36-year-old woman with no significant medical history, a homemaker and farmer, resident of a rural community in Oaxaca, Mexico. She was referred to the ENT Department of the General Hospital of Mexico with a history of a slow-growing painless nasal tumor with 1 year of evolution, associated with constant bilateral nasal obstruction that had increased since the onset of the disease. On questioning, the patient denied the presence of trauma, fever, weight loss, rhinorrhea, and
Correspondence:

*Carlos Santamaría-Saad

E-mail: carlos.santamaria93@gmail.com
Available online: $22-01-2021$

Date of reception: 20-02-2020

Date of acceptance: 29-06-2020 DOI: 10.24875/HGMX.20000034
Rev Med Hosp Gen Mex. 2021;84(1):32-35 www.hospitalgeneral.mx 0185-1063/@ 2020 Sociedad Médica del Hospital General de Mexico. Published by Permanyer. This is an open access article under the CC BYNC-ND license (http://creativecommons.org/licenses/by-nc-nd/4.0/). 


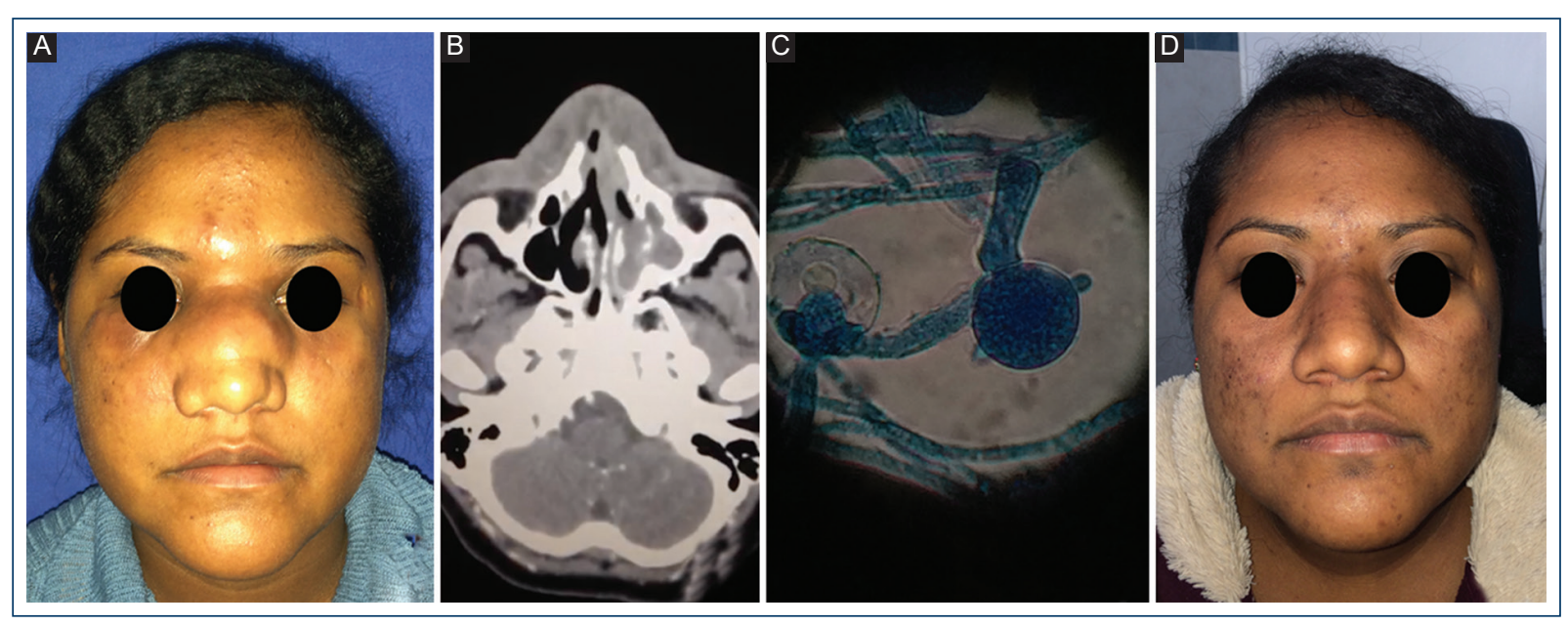

Figure 1. A: patient \#1 with nasal tumor of 1-year evolution. B: nasal tomography showing occupation of maxillary sinus, middle meatus, left ethmoid air cells and soft tissue infiltration. C: Classic appearance of Conidiobolus coronatus (broad pauciseptate hyphae). D: Evident clinical improvement after 6 months of treatment.

other associated nasal symptoms. Physical examination showed an increase in centrofacial volume that involved the frontal region, glabella, nasal dorsum, zygomatic arch, and infraorbital ridges (Fig. 1A). Patient was previously treated with unspecified antibiotic therapy, without improvement. It was decided to perform a contrasted tomography of the nose and paranasal sinuses, assessing the occupation of the middle meatus, maxillary sinus and left ethmoid air cells, as well as the infiltration of the soft tissues in the nasal dorsum, glabella, frontal, and periorbital regions (Fig. 1B). Nasal flexible endoscopy showed no changes in the appearance of the nasal mucosa. The glabella was biopsied in the operating theatre and was subjected to histopathological study, which reported fibrosis, with areas of inflammatory infiltration of lymphocytes, histiocytes, and multinucleated giant cells (MNGC) type "A," as well as foreign body. Direct examination with $20 \%$ potassium hydroxide showed broad, pauciseptate hyphae that branch irregularly at straight angles, surrounded by intense eosinophilic material (Fig. 1C). Mycological culture developed Conidiobolus coronatus. It was classified as a Stage I disease and was treated with trimethoprim (TMP)/sulfamethoxazole (SMZ) for 10 days and later 14 months of oral itraconazole at a reduced dose. Improvement was clinically evident at 6 months (Fig. 1D).

\section{Second case}

A 32-year-old woman, no significant medical history, working in the banking sector, resident in Mexico City, she was referred to our practice with a 20-day clinical condition regarding an intermittent bilateral nasal obstruction, with no predominant laterality, purulent anterior rhinorrhea, which was treated empirically with oral ciprofloxacin without improvement of symptoms. Later on, patient reported an increase in the centrofacial volume that involved the glabella and the nasal dorsum (Fig. 2A). The tomography showed increased density of the tissues of the nasal dorsum and non-specific mucosal thickening (Fig. 2B). The glabella was biopsied in the operating theatre and was subjected to mycological analysis, which revealed $C$. coronatus. Like the first patient, she was classified as a Stage I disease and was treated with TMP/SMZ for 10 days. She was later prescribed oral itraconazole at a reduced dose for 10 months. Improvement was clinically evident at 2 months (Fig. 2C).

\section{Discussion}

Conidiobolomycosis is a subcutaneous mycosis. Isolated in the soil and decomposed plants, it infects mites and fleas, and subsequently mammals such as chimpanzees and horses ${ }^{1}$. In humans, it is suspected that infection occurs by direct trauma or inhalation of spores $^{2}$. It has an unknown incubation period and characteristically affects the rhinofacial region with slow, progressive, and painless subcutaneous and submucosal inflammation ${ }^{3}$.

The condition typically manifests itself in healthy, middle-aged adults hailing from tropical regions 


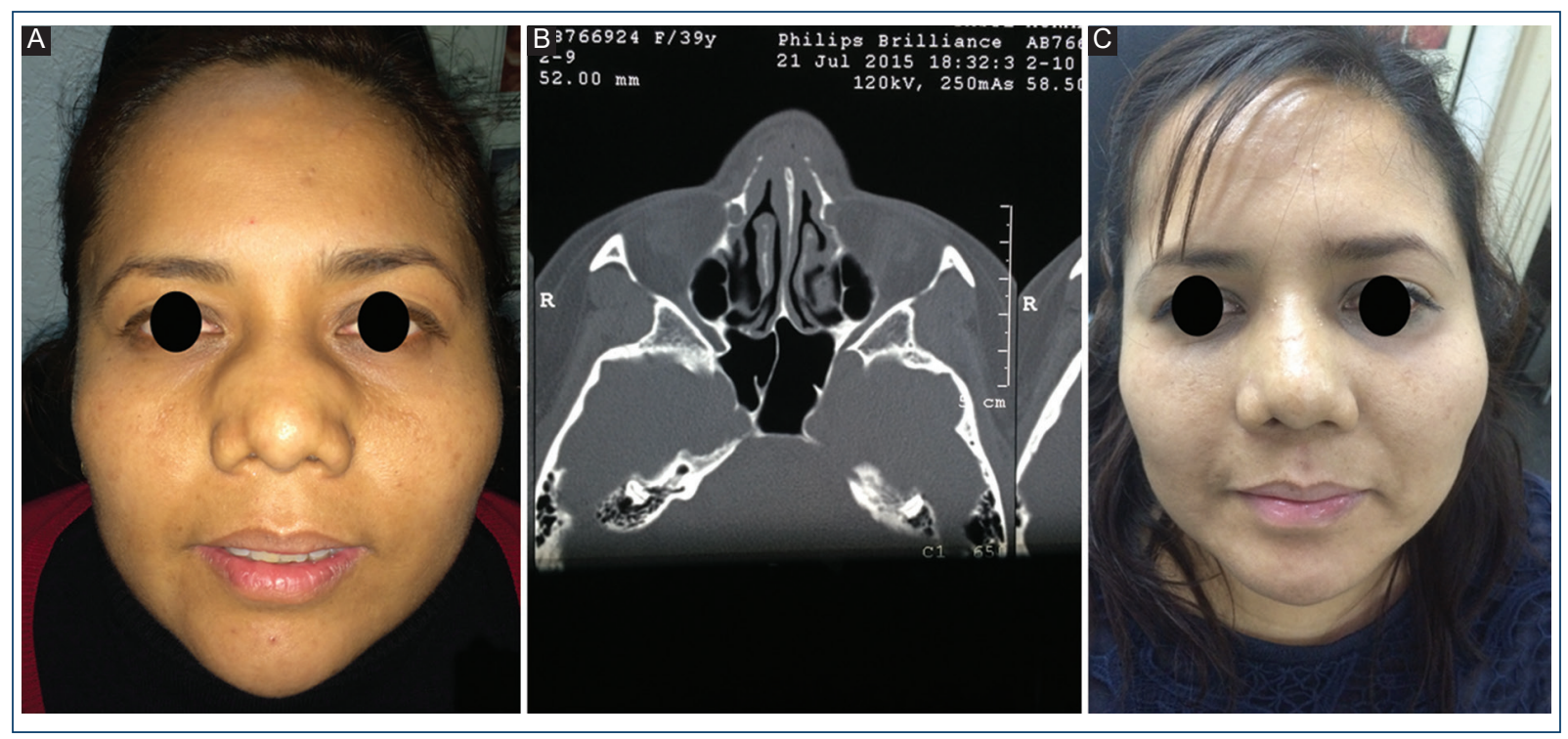

Figure 2. A: Patient \#2 with a 20-day tumor on the nasal dorsum and glabella. B: Nasal tomography with soft-tissue infiltration and mucosal thickening C: Evident clinical improvement after 2 months of treatment.

worldwide ${ }^{3}$. It seldom occurs in patients living in urban areas such as the patient featured in clinical case number 2 , as it is related to the presence of spores in rural areas with a tropical climate. On average, they occur after 10 months of progressive nasal symptoms, edema, or facial swelling. It can also lead to facial elephantiasis, due to lymphatic congestion, producing serious facial malformations that require extensive surgical treatments ${ }^{4}$. In rare cases, usually associated with immunocompromised patients, it manifests itself in an atypical way, in different regions other than the face. In the most serious and rare cases, it manifests itself in a systemic way, affecting the lower respiratory tract $^{5}$.

The clinical classification of conidiobolomycosis includes three stages: Stage I: involvement limited to nostrils, paranasal sinuses, and pharynx; Stage II: spreads to the frontal region and/or the lips; and Stage III: affects muscles, bones, and viscera. In case of a suspected condition, biopsy should be performed with simple staining that is within the reach of many hospital centers. The fungus structures can be easily identified with histochemical staining, such as hematoxylin, Periodic acid-Schiff or Grocott-Gomori's Methenamine Silver ${ }^{2}$. Although it requires an assessment by mycology experts familiar with the pathology, an initial biopsy where a suppurative granuloma is seen with lymphocytes, histiocytes, MNGC, plasma cells, and eosinophils, could hint at a first approach to the diagnosis. Sometimes pauciseptate or non-septate hyphae are seen surrounded by an eosinophilic halo (Splendore-Hoeppli phenomenon), but this does not distinguish between Zygomycetes species $^{6,7}$.

It is important to consider other causes of benign and malignant tumors of the nasal and centrofacial region as differential diagnoses, as well as sarcoidosis and rhinosporidiosis ${ }^{2}$. There are no established guidelines for treatment. However, monotherapy with antifungal (potassium iodide, fluconazole, ketoconazole, amphotericin B, and itraconazole) or antibacterial (TMP-SMZ) drugs in cases of localized disease has proven to be sufficient ${ }^{8,9}$. Based on international literature, our patients were treated empirically with initial doses of TMP-SMZ and itraconazole orally for a long time. Both drugs have been shown to be active against C. coronatum ${ }^{9}$, the duration of which was $>10$ months and reduced the likelihood of persistent disease ${ }^{1}$. In cases of atypical, disseminated or complicated disease, therapies with two, three and up to four antimicrobials should be considered ${ }^{10}$. Facial esthetic complications should be treated with debridement and surgical reconstruction 2,10 .

\section{Conflicts of interest}

The authors declare no conflicts of interest. 


\section{Ethical disclosures}

Protection of human and animal subjects. The authors declare that no experiments were performed on humans or animals for this study.

Confidentiality of data. The authors declare that they have followed the protocols of their work center on the publication of patient data.

Right to privacy and informed consent. The authors have obtained the written informed consent of the patients or subjects mentioned in the article. The corresponding author is in possession of this document.

\section{References}

1. Kimura M, Yaguchi T, Sutton D, Fothergill A, Thompson E, Wickes B. Disseminated human conidiobolomycosis due to conidiobolus lamprauges. J Clin Microbiol. 2011;49:752-56.
2. Isa-Isa R, Arenas R, Fernández R, Isa M. Rhinofacial conidiobolomycosis (entomophthoramycosis). Clin Dermatol. 2012;30:409-12.

3. Yang X, Li Y, Xhou X, Wang Y, Geng S, Liu H, et al. Rhinofacial conidiobolomycosis caused by Conidiobolus coronatus in a Chinese rice farmer. Mycoses. 2009; 53:369-73.

4. Choon S, Kang J, Neafie R, Ragsdale B, Klassen-Fischer M, Carlson J. Conidiobolomycosis in a young Malaysian woman showing chronic localised fibrosing leukocytoclastic vasculitis: a case report and meta-analysis focusing on clinicopathologic and therapeutic correlations with outcome. Am J Dermatopathol. 2012;34:511-22.

5. Twizeyimana E, Chauty A, Cihet M, Ardant M, Adeye A, Zidane M, et al. Rhinofacial conidiobolomycosis associated with cervical, thoracic and branchial localisations: one clinical case in Nigeria. J Mycol Med. 2014;24:48-55.

6. Sharma N, Mahajan V, Singh P. Orofacial conidiobolycosis due to Conidiobolus incongruus. Mycoses. 2003;46:137-40.

7. Kaufman L, Mendoza L, Standard P. Immunodiffusion test for serodiagnosing subcutaneous zygomicosis. J Clin Microbiol. 1990;28:1887-90.

8. Juliana T, Loreto E, Jesus F, Dutra V, Nakazato L, Alves S, et al. In vitro assessment of antifungal drugs and sulfamethoxazole-trimethoprim against clinical isolates of Conidiobolus lamprauges. Antimicrob Agents Chemother. 2018:62:1-4.

9. Gupta M, Narang T, Kaur RJ, Manhas A, Sairia U, Dogra S. A prospective case series evaluating efficacy and safety of combination of itraconazole and potassium iodide in rhinofacial conidiobolomycosis. Int $\mathrm{J}$ Dermatol. 2016;55:208-14.

10. Radhakrishnan N, Sachdeva A, Oberoi J, Prakash S. Conidiobolomycosis in relapsed acute lymphoblastic leukemia. Pediatr Blood Cancer. 2009;53:1321-3. 